\title{
Evaluación de competencias docentes de instituciones públicas mexicanas de nivel medio superior
}

\author{
Ruiz Gil Yakeline $^{\text {a }}$, Ortiz Espinoza Jesús ${ }^{\text {b }}$, Carreto Bernal Fernando ${ }^{c}$ \\ ${ }^{a}$ Universidad Autónoma del Estado de México, Estado de México. yakyruiz13@gmail.com, \\ ${ }^{\mathrm{b}}$ Universidad Iberoamericana, Ciudad de México, México. ether_azul@yahoo.com.mx, ${ }^{\mathrm{c}}$ Universidad \\ Autónoma del Estado de México, Estado de México. fcarretomx@yahoo.com.mx
}

\section{Resumen}

En la primera década del siglo XXI, en México, se ha intentado implementar un modelo educativo distinto al tradicional, conocido como "modelo de educación basada en competencias" en respuesta a las necesidades laborales que origina la sociedad del conocimiento y/o de la información. Diversas investigaciones se han centrado en observar los procesos de evaluación, el desempeño, el análisis del proceso de enseñanza-aprendizaje de los docentes desde diversas perspectivas; sin embargo, en este trabajo se aborda el análisis de las competencias docentes de forma cualitativa $y$ cuantitativa para el nivel medio superior desde la propuesta de la Reforma Integral de la Educación Media Superior acuerdo 447 publicadas en el Diario Oficial de la Federación en el año 2008. El estudio realizado involucra la comparación de dos unidades de académicas de la Universidad Autónoma del Estado de Guerrero, UAGro. El instrumento de evaluación empleado fue un cuestionario de 98 reactivos con una escala tipo likert constituyendo un inventario y diagnóstico acerca de las competencias docentes con que cuentan los profesores del Nivel Medio Superior. Se encuentra estructurado en seis ejes interdisciplinarios: los saberes pedagógicos; organización de la enseñanza; comunicación; interacción; intervención pedagógica; y desarrollo profesional. Los resultados evidencian que los docentes emplean en mayor proporción la tecnología para desempeñar sus actividades profesionales, es decir, su estilo de enseñanza se basa en el uso de las TIC fortaleciendo sus quehaceres educativos. En general, las habilidades más valoradas y utilizadas por los docentes son saberes pedagógicos e interacción social y las menos valoradas son comunicación, interacción psicopedagógica.

Palabras claves: competencias docentes, evaluación de competencias, ejes interdisciplinarios. 


\section{Introducción}

En la primera década del siglo XXI en México se han intentado implementar un modelo educativo distinto al tradicional, conocido como "modelo de educación basada en competencias" con el objetivo de generar habilidades en los estudiantes que pudieran corresponder a las demandas sociales como individuales del presente siglo, como lo señala Prrenoud (2007) una competencia es cuando se emplea las capacidades o aptitudes para hacer frente a las situaciones que se presente. Este aspecto se vincula a los procesos laborales que fundamentados en las demandas del trabajo.

Esta vinculación entre el sector laboral y el sector productivo ha permitido que se capte con mayor claridad las necesidades reales de la sociedad, (ANUIES: 2003, p.31) favoreciendo de esta manera la búsqueda de soluciones a los problemas que se presentan tanto en la sociedad como en el sector productivo, redefiniendo las políticas educativas. Yolanda Argudín señala que el concepto de competencias en la educación significa básicamente "saberes de ejecución" ya que todo proceso de "conocer" se traduce en un "saber" (Argudín: 2005, p.3). Por otro lado Tobon (2006) menciona que las competencias son procesos complejos en el desesempeño que corresponden al contexto con responsabilidad.

La educación por competencias pretende vincular el sector educativo con el sector productivo, a fin de elevar el potencial de los individuos, frente a la sociedad contemporánea (Cejas, p. 4). Así como para cumplir con las demandas del siglo XXI el docente como parte elemental de la educación vanguardista, deberá poseer conocimientos académicos, administrativos y humano-sociales (Aldape, 2008).

De acuerdo a Magda Cejas, el concepto de competencias vinculada a la educación es una combinación de aplicación de conocimientos, habilidades o destrezas en la realización de un trabajo, se puede expresar: El saber, El saber hacer y El saber ser. De tal manera que se llega a las siguientes consideraciones: La formación por competencias debe ir más allá de transmitir saberes y destrezas manuales, por lo que debe buscar incrementar la capacidad de las personas, además de considerar aspectos culturales, sociales y actitudinales.

En el acuerdo 447, publicada en el DOF el día miércoles 29 de octubre de 2008, se define el perfil del docente del Sistema Nacional de Bachillerato (SNB), y se establece las competencias que deberá cumplir los docentes de las instituciones educativas que en la modalidad escolarizada imparten educación de nivel medio superior y operen en el Sistema Nacional de Bachillerato. 
Con regularidad se delega a la institución el diseño y evaluación de la evaluación, sin embargo, no se tienen directrices para evaluar el desempeño docente, o la evaluación es manejada por la opinión de los estudiantes, a través de cuestionarios aplicados por los jefes inmediatos o autoevaluaciones a profesores. Por ello, se propuso contribuir a través de este proyecto, una evaluación cuantitativa y cualitativa de los niveles de desarrollo de las competencias docentes de los profesores de las Unidades académicas 23 y 35 de la Universidad Autónoma de Guerrero.

\section{Materiales y Métodos}

Para elaborar esta investigación fue necesario analizar los programas de capacitación y actualización implementados en el Nivel Medio Superior. Además, se organizó la información estableciendo relaciones, similitudes y diferencias sobre la problemática abordada, su instrumentación y resultados, para lo cual se empleo como instrumento de evaluación el cuestionario de evaluación de competencias docentes (CECD) que constituye un inventario y diagnóstico acerca de las competencias con que cuentan los docentes. El estudio se realizó en las Unidades Académicas No. 23 y 35 de la UAG ubicadas en la Región Costa Grande del Estado de Guerrero. Principalmente se utilizó el cuestionario de evaluación de competencias docentes, elaborado por Dávila y Vaca (2012), para conocer el estado actual que guardan los profesores de la región enunciada con respecto a sus competencias docentes en la enseñanza de un área especifica.

Este cuestionario contiene 98 reactivos con descripciones sobre las competencias que deben utilizar los profesores. Cada reactivo incluye una escala con un intervalo de variadas opciones tipo likert, a fin de facilitar y homogeneizar las respuestas de los profesores.Las áreas y ejes que explora el cuestionario de evaluación de las competencias docentes son las siguientes: saberes pedagógicos; organización de la enseñanza; comunicación; interacción social; intervención psicopedagógica; y desarrollo profesional.

El procedimiento en la investigación en primera instancia fue caracterizar las competencias mínimas y las deseables, se realizó el estudio del arte del tema y el análisis de documentos oficiales de la Universidad Autónoma de Guerrero, así como la consulta en las unidades Académicas involucradas. De este análisis se formuló una primera versión de las competencias que deben tener los profesores sobre la base de los aspectos antes descritos., siendo evaluada por los investigadores y los propios profesores para integrar la versión definitiva. Posteriormente se llevó a cabo la aplicación de los cuestionarios de evaluación de las competencias docentes (CECD) a la muestra de población seleccionada de las 
unidades académicas 23 y 35. Finalmente se recabo la información para llevar a cabo la representación gráfica, la descripción y análisis de resultados.

\section{Resultados}

\section{Unidad académica 23}

En la figura 1 se presentan los seis ejes en los que se evaluaron a los docentes de la UA23, inicialmente, el eje de comunicación, al que le corresponden los atributos de lectura; escritura; audición y expresión oral, representa un promedio del 32\% de la competencia, considerando que el valor es inferior al $50 \%$ en el dominio de la comunicación. El atributo más valorado por los docentes es la expresión oral y el menos valorado es la escritura. Se considera como valor mínimo aceptable el $60 \%$ de dominio de las habilidades, sin embargo, los resultados están por debajo de éste. En el área de desarrollo profesional, se consideraron dos atributos: formación permanente y conciencia crítica, alcanzando en promedio $42 \%$ de dominio de los dos atributos, considerando que en la formación permanente es más alto el dominio frente a la conciencia crítica del eje valorado.

En el área de la intervención psicopedagógica, los atributos analizados son las estrategias y formas de trabajo resultando en promedio un $35 \%$ de dominio del área. Lo que permite inferir que los docentes no emplean diversas estrategias didácticas y que no cuentan con distintas actividades para los momentos de la clase. Carecen de técnicas de la enseñanza y del aprendizaje. En los saberes pedagógicos, los atributos involucrados en esta área son: las concepciones acerca de los adolescentes, el uso de las TIC, el pensamiento lógico y la explicación de la realidad social, en donde el promedio del área es de $43 \%$ de dominio. Sin embargo, el atributo con más aplicación por parte de los docentes es el uso de las TIC y la realidad social, mientras que el atributo menos valorado en este eje es la concepción de la adolescencia.

En la organización de la enseñanza, se evaluaron dos atributos: la planeación y la evaluación, que en promedio representa $29 \%$ de dominio, valorando la planeación en sus actividades profesionales y enfocándose en los contenidos que el alumno debe conocer, pero omitiendo los indicadores de las competencias que se encuentran inmersos dentro del proceso de desarrollo de los estudiantes. Finalmente, el área de interacción social, que involucra el atributo de la convivencia, tiene un dominio del $30 \%$ denotando poca disponibilidad en el trabajo colaborativo y poco compromiso con la institución. En términos generales la UA 23 muestra que sus docentes se encuentran deficientes en el conocimiento, aplicación y dominio de las competencias que el SNB requiere para que los alumnos cumplan con el perfil de egreso requerido. 
Los resultados obtenidos de la aplicación del cuestionario en la Unidad Académica 23 (UA23) se muestran en la figura 1.

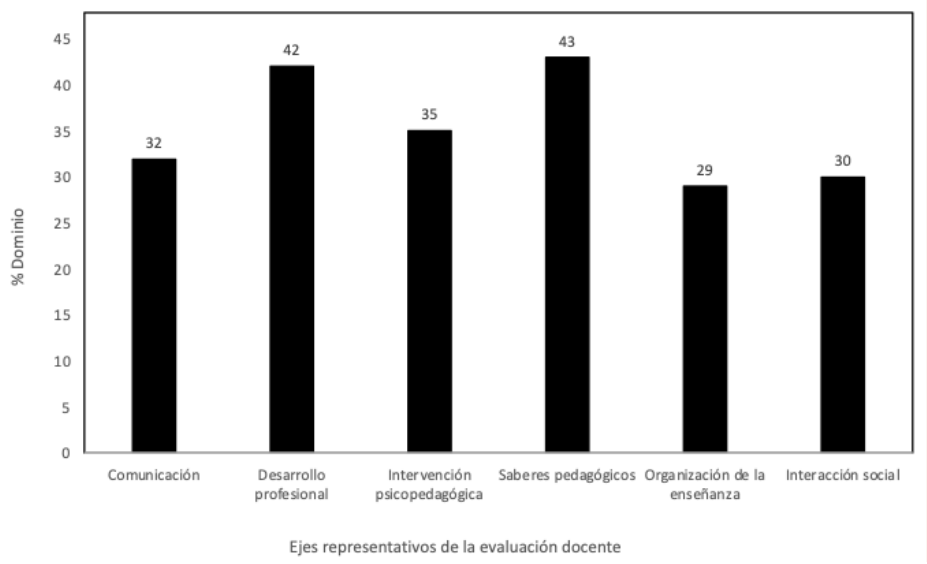

Fig 1. Representación por áreas de evaluación de las competencias de los docentes de la UA 23.

\section{Unidad Académica 35}

En la figura 2 se muestran los seis ejes en los que se evaluaron a los docentes de la UA35, en el primer eje de evaluación esta la comunicación, que obtuvo un promedio de 32\%, resaltando que la audición fue el valor que predomina, seguido de la expresión oral y que el atributo menos valorado fue la escritura, especificando que éste último fue igual para la UA23.

En el área de desarrollo profesional, se obtuvo un 44\%, destacando que el atributo más valorado por la UA35 fue el de formación permanente y el menos valorado es el de conciencia crítica. Demostrando que los docentes se encuentran en constante actualización, sin embargo, no son lo suficientemente altos para indicar que cubren las especificaciones solicitadas por el Marco Curricular de las competencias docentes.El área de intervención psicopedagógica, fue de un $38 \%$ de dominio del área en promedio, sin embargo, este valor se encuentra por debajo de lo requerido.

En los saberes pedagógicos se obtuvo un 53\% resaltando que es el promedio más representativo de los ejes evaluados en ambas Unidades Académicas, no obstante, el atributo que más dominan los docentes es el uso de las TIC, lo que implica que el empleo de la tecnología es la herramienta que más utilizan para sus actividades académicas, seguido del pensamiento lógico y el menos estimado fue la concepción de la adolescencia. 
En la organización de la enseñanza, se logró un 37\% de dominio del eje, resaltando la planeación, por encima de la evaluación con una diferencia mínima, involucrando la planeación en sus actividades profesionales y la evaluación como parte de la misma. Finalmente, en la evaluación del área de interacción social, posee en promedio un dominio del $47 \%$ denotando que los docentes se involucran en la comunidad educativa, sin embargo, no es suficiente y se requiere mayor participación y compromiso con sus homólogos y padres de familia.

Los resultados obtenidos de la aplicación del cuestionario en la Unidad Académica 35 (UA35) se muestran en la figura 2.

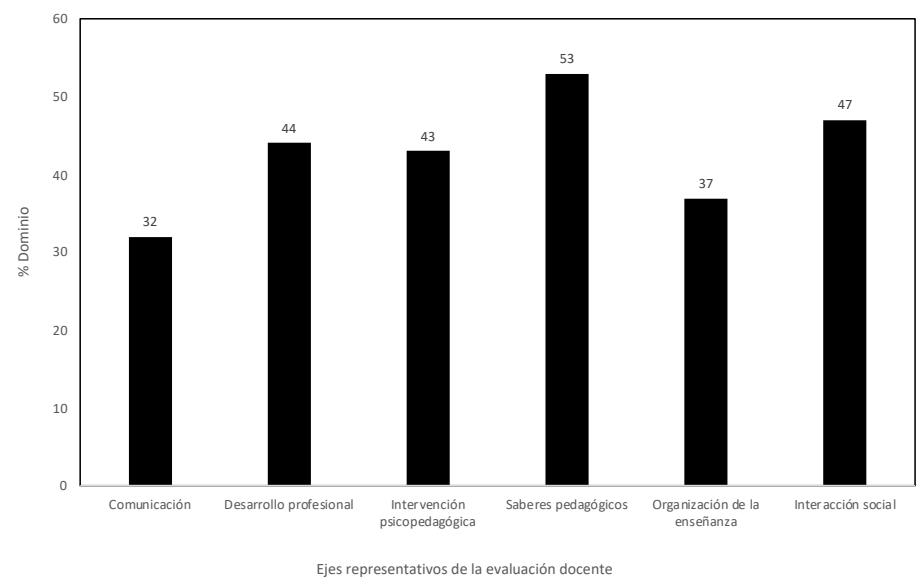

Fig. 2. Representación por áreas de evaluación de las competencias de la UA 35.

\section{Discusión}

En la Tabla 1 se observan, de manera resumida, los ejes evaluados de la UA 23 y UA 35. El valor más representativo son los saberes pedagógicos, lo que implica, la recuperación de los conocimientos acerca de la adolescencia, el cuidado de la salud física y mental, las relaciones con el medio natural, la explicación de la realidad, el aprovechamiento de la tecnología y el pensamiento lógico matemático. En el valor menos valorado por las UA es el eje de la organización de la enseñanza en la UA23 mientras que para la UA35 es la comunicación, considerando que el promedio se encuentra en un porcentaje inferior al 50\%, destacando que estas habilidades son básicas en la formación básica del estudiante. 
Los resultados muestran que los docentes emplean en mayor medida la tecnología para desempeñar sus actividades profesionales, su estilo de enseñanza se basa en el uso de las TIC. Algunos rubros con menor aprovechamiento son por ejemplo, el eje de la organización enseñanza de la UA23 en cuanto a la evaluación con un porcentaje del 26\%, asimismo, la escritura con un $27 \%$ en ambas Unidades.

Tabla 1. Competencias docentes de acuerdo a los ejes de evaluación.

\begin{tabular}{|c|c|c|c|c|c|c|c|}
\hline \multirow{2}{*}{$\begin{array}{c}\text { Ejes de } \\
\text { evaluación }\end{array}$} & \multicolumn{4}{|c|}{ Comunicación } & \multicolumn{2}{|c|}{ Desarrollo profesional } & \multirow{2}{*}{$\begin{array}{c}\text { Intervención } \\
\text { psicopedagógica }\end{array}$} \\
\hline & Lectura & Escritura & Audición & $\begin{array}{c}\text { Expresión } \\
\text { oral }\end{array}$ & $\begin{array}{l}\text { Formación } \\
\text { permanente }\end{array}$ & $\begin{array}{c}\text { Conciencia } \\
\text { crítica }\end{array}$ & \\
\hline $\begin{array}{c}\text { Valores de } \\
\text { dominio }\end{array}$ & 32 & 27 & 34 & 36 & 44 & 40 & 35 \\
\hline UA23 & \multicolumn{4}{|c|}{32} & \multicolumn{2}{|c|}{42} & 35 \\
\hline $\begin{array}{c}\text { Valores de } \\
\text { dominio }\end{array}$ & 32 & 27 & 34 & 36 & 49 & 38 & 43 \\
\hline UA35 & \multicolumn{4}{|c|}{32} & \multicolumn{2}{|c|}{44} & 43 \\
\hline \multirow{2}{*}{$\begin{array}{c}\text { Ejes de } \\
\text { evaluación }\end{array}$} & \multicolumn{4}{|c|}{ Saberes pedagógicos } & \multicolumn{2}{|c|}{$\begin{array}{c}\text { Organización de la } \\
\text { enseñanza }\end{array}$} & Interacción social \\
\hline & Adolescencia & $\begin{array}{c}\text { Realidad } \\
\text { Social } \\
\end{array}$ & Tecnología & $\begin{array}{c}\text { Pensamiento } \\
\text { lógico }\end{array}$ & Planeación & Evaluación & Convivencia \\
\hline $\begin{array}{c}\text { Valores de } \\
\text { dominio }\end{array}$ & 31 & 44 & 58 & 38 & 31 & 26 & 30 \\
\hline UA23 & \multicolumn{4}{|c|}{43} & \multicolumn{2}{|c|}{29} & 30 \\
\hline $\begin{array}{c}\text { Valores de } \\
\text { dominio }\end{array}$ & 42 & 48 & 73 & 49 & 38 & 36 & 47 \\
\hline UA35 & \multicolumn{4}{|c|}{53} & \multicolumn{2}{|c|}{37} & 47 \\
\hline
\end{tabular}

\section{Conclusiones}

Para lograr el perfil educativo solicitado el docente debe colaborar en la construcción de proyectos de formación integral dirigido a los estudiantes en forma colegiada con otros docentes y los directivos de la institución, así como el personal de apoyo técnico pedagógico. En general, los resultados de la evaluación de las competencias docentes de las unidades académicas 23 y 35 evidencian que, en la ctualidad, el uso de la tecnología es la herramienta que más utilizan para sus actividades académicas, seguido del pensamiento lógico y el menos estimado fue la concepción de la adolescencia.

Es importante destacar que los resultados obtenidos de esta investigación arrojaron diferencias significativas entre el dominio de las competencias docentes de las dos unidades 
académicas atribuibles a la falta de actualización continua e innovaciones estratégicas en el desarrollo profesional.

El porcentaje de dominio de los diferentes ejes evaluados para ambas unidades académicas se encuentran por debajo del valor minimo aceptable (60\%) evidenciando que los docentes se encuentran deficientes en el conocimiento, aplicación y dominio de las competencias que el Sistema Nacional de Bachillerato requiere para que los alumnos cumplan con el perfil de egreso requerido.

\section{Referencias}

Aldape, T. (2008) Desarrollo de las competencias del docente. Siglo XXI, México.

ANUIES. (2003) La educación superior en el siglo XXI. ANUIES, México.

Argudín, Y. (2005). Educación basada en competencias. Nociones y antecedentes. México: Trillas.

Cejas, M. La Educación basada en competencias: una metodología que impone en la Educación Superior y que busca estrechar la brecha existente entre el sector educativo y el productivo.

Dávila, E., Vaca, Y. (2012). Hacia una Evaluación participativa y pública. Revista electrónica Multidisciplinaria Aprendizaje-REMA.

Perrenoud, Ph. (2007). Diez nuevas competencias para enseñar. Cuarta edición. Barcelona, Graó.

Tobón, S. (2004). Formación basada en competencias: Pensamiento complejo, diseño curricular y didáctica. Bogotá: ECOE.

SEMS (2008). Competencias que expresan el perfil del docente de la Educación Media Superior, Documento interno. Subsecretaría de educación Media Superior. México: SEP. 